GANIT J. Bangladesh Math. Soc. (ISSN 1606-3694) 34 (2014) 35-46

\title{
JORDAN DERIVATIONS ON LIE IDEALS OF SEMIPRIME $\Gamma$-RINGS
}

\author{
Md. Mizanor Rahman ${ }^{1}$ and Akhil Chandra Paul ${ }^{2}$ \\ ${ }^{1}$ Department of Mathematics, Jagannath University, Dhaka-1100, Bangladesh \\ ${ }^{2}$ Department of Mathematics, University of Rajshahi, Rajshahi-6205, Bangladesh \\ ${ }^{1}$ E-mail: acpaulrubd_math@yahoo.com; ${ }^{2}$ E-mail: mizanorrahman@gmail.com
}

Received 05.05.2014

Accepted 07.12.2014

\begin{abstract}
Let $M$ be a 2-torsion free semiprime $\Gamma$-ring satisfying the condition $a \alpha b \beta c=a \beta b \alpha c, \forall a, b, c \in M$ and $\alpha, \beta \in \Gamma$. Let $U$ be an admissible Lie ideal of $M$ that is, $u \alpha u \in U, \forall u \in U, \alpha \in \Gamma$ and $U \subseteq Z(M)$, the centre of $M$. If $\mathrm{d}: M \rightarrow M$ is an additive mapping such that $d$ is a Jordan derivation on $U$ of $M$, then $d$ is a derivation on $U$.
\end{abstract}

Keywords: Derivation, Jordan derivation, Lie ideal, admissible Lie ideal, $\Gamma$-ring, semiprime $\Gamma$-ring

\section{Introduction}

Let $M$ and $\Gamma$ be additive abelian groups. If there is a mapping $M \times \Gamma \times M \rightarrow M$ (sending $(x, \alpha, y)$ into $x \alpha y)$ such that

- $(x+y) \alpha z=x \alpha z+y \alpha z, x(\alpha+\beta) y=x \alpha y+x \beta y, x \alpha(y+z)=x \alpha y+x \alpha z$

- $(x \alpha y) \beta z=x \alpha(y \beta z), \forall x, y, z \in M$ and $\alpha, \beta \in \Gamma$

then $M$ is called a $\Gamma$-ring. This concept is more general than a ring and was introduced by Barnes [3]. A $\Gamma$-ring $M$ is called a prime $\Gamma$-ring if $\forall a, b \in M, a \Gamma M \Gamma b=0$ implies $a=0$ or $b=0$ and $M$ is called semiprime if $a \Gamma M \Gamma a=0$ (with $a \in M$ ) implies $a=0$. A $\Gamma$-ring $M$ is 2 -torsion free if $2 a=0$ implies $a=0, \forall a \in M$. For any $x, y \in M$ and $\alpha \in \Gamma$, we denote the commutator $x \alpha y-y \alpha x$ by $[x, y]_{\alpha}$ An additive subgroup $U \subset M$ is said to be a Lie ideal of $M$ if whenever $u \in U, m \in M$ and $\alpha \in \Gamma$, then $[u, m]_{\alpha} \in U$. In the main results of this article we assume that the Lie ideal $U$ verifies $u \alpha u \in U, \forall u \in U, \alpha \in \Gamma$. A Lie ideal of this type is called a square closed Lie ideal. Furthermore, if the Lie ideal $U$ is square closed and $U \nsubseteq Z(M)$, where $Z(M)$ denotes the center of $M$ then $U$ is called an admissible Lie ideal of $M$. Let $M$ be a $\Gamma$-ring. An additive mapping $d: M \rightarrow M$ is a derivation if $d(a \alpha b)=d(a) \alpha b+a \alpha d(b)$ and a Jordan derivation if $d(a \alpha a)=d(a) \alpha a+a \alpha d(a), \forall a, b \in M$ and $\alpha \in \Gamma$. Throughout the article, we use the condition $a \alpha b \beta c=a \beta b \alpha c, \forall a, b, c \in M$ and $\alpha, \beta \in \Gamma$ and this is denoted by $(*)$. The relationship between usual derivations and Lie ideals of prime rings has been extensively studied in the last forty years. In particular, when this relationship involves the action of the derivations on Lie ideals. In 1984, R. Awtar [2] extended to Lie ideals a 
well known result proved by I. N. Herstein [10] which states that 'every Jordan derivation on a 2-torsion free prime ring is a derivation'. In fact, R. Awtar [2] proved that if $U \nsubseteq$ $Z(M)$ is a square closed Lie ideal of a 2-torsion free prime ring $R$ and $d: R \rightarrow R$ is an additive mapping such that $d\left(u^{2}\right)=d(u) u+u d(u), \forall u \in U$, then $d(u v)=d(u) v+u d(v)$, $\forall u, v \in U$. M. Ashraf and N. Rehman [1] studied on Lie ideals and Jordan left derivations of prime rings. They proved that if $d: R \rightarrow R$ is an additive mapping on a 2-torsion free prime ring $R$ satisfying $d\left(u^{2}\right)=2 u d(u), \forall u \in U$, where $U$ is a Lie ideal of $R$ such that $u^{2} \in U, \forall u \in U$, then $d(u v)=d(u) v+u d(v), \forall u, v \in U$. A. K. Halder and A. C. Paul [9] extended the results of Y. Ceven [6] in Lie ideals. We have generalized the R. Awtar's [2] result in semiprime $\Gamma$-rings.

\section{Jordan Derivation on a Lie Ideal}

Definition 2.1 Let $M$ be $a \Gamma$-ring and $U$ be a Lie ideal of $M$. An additive mapping $d: M \rightarrow M$ is said to be a Jordan derivation on a Lie ideal $U$ of $M$ if $d(u \alpha u)=d(u) \alpha u+u \alpha d(u), \forall u \in U$ and $\alpha \in \Gamma$.

Example 2.1 Let $R$ be a ring of characteristic 2 having a unity element 1 .

Let $M=M_{1,2}(R)$ and $\Gamma=\left\{\left(\begin{array}{c}n .1 \\ n .1\end{array}\right): n \in \mathbb{Z}, 2 \nmid n\right\}$.

Then $M$ is a $\Gamma$-ring. Let $N=\{(x, x): x \in R\} \subseteq M$.

Now $\forall(x, x) \in N,(a, b) \in M$ and $\left(\begin{array}{l}n \\ n\end{array}\right) \in \Gamma$, we have

$(x, x)\left(\begin{array}{l}\mathrm{n} \\ \mathrm{n}\end{array}\right)(a, b)-(a, b)\left(\begin{array}{l}n \\ n\end{array}\right)(x, x)=(x n a-b n x, x n b-a n x)$

$=(x n a-2 b n x+b n x, b n x-2 a n x+x n a=(x n a+b n x, b n x+x n a) \in N$.

Therefore, $N$ is a Lie ideal of $M$.

Example 2.2 Let $M$ be a $\Gamma$-ring satisfying the condition (*) and let $U$ be a Lie ideal of $M$. Let $a \in M$ and $\alpha \in \Gamma$ be fixed elements. Define $d: M \rightarrow M$ by $d(x)=a \alpha x-x \alpha a, \forall x \in U$. Now $\forall y \in U$ and $\beta \in \Gamma$, we have $d(x \beta y)=a \alpha x \beta y-x \beta y \alpha a$, $=(a \alpha x-x \alpha a) \beta y+$ for every $x, y \in U$ and $\beta \in \Gamma$. Therefore $d: M \rightarrow M$ is a derivation on $U$.

Example 2.3 Let $M$ be $a \Gamma$-ring and $U$ be a Lie ideal of $M$. Let $d: M \rightarrow M$ be a derivation on $U$. Suppose $M_{1}=\{(x, x): x \in M\}$ and $\Gamma_{1}=\{(\alpha, \alpha): \alpha \in \Gamma\}$. Define addition and multiplication on $M_{1}$ as $(x, x)+(y, y)=(x+y, x+y)$ and $(x, x)(\alpha, \alpha)(y, y)=(x \alpha y, x \alpha y)$. Then $M_{1}$ is a $\Gamma_{1}$-ring. Suppose $U_{1}=\{(u, u): u \in U\}$. Now $(u, u)(\alpha, \alpha)(x, x)-(x, x)(\alpha, \alpha)(u, u)=(u \alpha x, u \alpha x)-(x \alpha u, x \alpha u)=(u \alpha x-x \alpha u, u \alpha x-x \alpha u) \in U_{1}$ for $u \alpha x-x \alpha u \in U$. Hence $U_{1}$ is a Lie ideal of $M_{1}$. If we define a mapping 
$D: M_{1} \rightarrow M_{1}$ on $U_{1}$ by $D((u, u))=(d(u), d(u))$. Then it is clear that $D$ is a Jordan derivation on $U_{1}$ which is not a derivation on $U_{1}$.

Lemma 2.1 Let $M$ be $a$ r-ring and $U$ be a Lie ideal of $M$ such that $u \alpha u \in U, \forall u \in U$ and $\alpha \in \Gamma$. If $d$ is a Jordan derivation on $U$ of $M$, then $\forall a, b, c \in U$ and $\alpha, \beta \in \Gamma$, the following statements hold:

(i) $\quad d(a \alpha b+b \alpha a)=d(a) \alpha b+d(b) \alpha a+a \alpha d(b)+b \alpha d(a)$.

(ii) $\quad d(a \alpha b \beta a+a \beta b \alpha a)=d(a) \alpha b \beta a+d(a) \beta b \alpha a+a \alpha d(b) \beta a+a \beta d(b) \alpha a$ $+a \alpha b \beta d(a)+a \beta b \alpha d(a)$ In particular, if $M$ is 2-torsion free and satisfies the condition $(*)$, then

(iii) $d(a \alpha b \beta a)=d(a) \alpha b \beta a+a \alpha d(b) \beta a+a \alpha b \beta d(a)$.

(iv) $d(a \alpha b \beta c+c \alpha b \beta a)=d(a) \alpha b \beta c+d(c) \alpha b \beta a+a \alpha d(b) \beta c+c \alpha d(b) \beta a+a \alpha b \beta d(c)$ $+c \alpha b \beta d(a)$.

Proof. Since $U$ is a Lie ideal satisfying the condition $a \alpha a \in U, \forall a \in U, \alpha \in \Gamma$. For $a, b \in U, \alpha \in \Gamma,(a \alpha b+b \alpha a)=(a+b) \alpha(a+b)-(a \alpha a+b \alpha b)$ and so $(a \alpha b+b \alpha a) \in U$.

Also, $[a, b]_{\alpha}=a \alpha b-b \alpha a \in U$ and it follows that $2 a \alpha b \in U$.

Hence $4 a \alpha b \beta c=2(2 a \alpha b) \beta c \in U, \forall a, b, c \in U, \alpha, \beta \in \Gamma$.

Thus

$$
\begin{aligned}
& d(a \alpha b+b \alpha a)=d((a+b) \alpha(a+b)-(a \alpha a+b \alpha b)) \\
& =d(a+b) \alpha(a+b)+(a+b) \alpha d(a+b)-d(a) \alpha a-a \alpha d(a)-d(b) \alpha b-b \alpha d(b) \\
& =d(a) \alpha a+d(a) \alpha b+d(b) \alpha a+d(b) \alpha b+a \alpha d(a)+a \alpha d(b)+b \alpha d(a)+b \alpha d(b)- \\
& d(a) \alpha a-a \alpha d(a)-d(b) \alpha b-b \alpha d(b)=d(a) \alpha b+a \alpha d(b)+d(b) \alpha a+b \alpha d(a) .
\end{aligned}
$$

Replacing $a \beta b+b \beta a$ for $b$ in (i) we get

$$
\begin{aligned}
& d(a \alpha(a \beta b+b \beta a)+(a \beta b+b \beta a) \alpha a) \\
& =d(a) \alpha(a \beta b+b \beta a)+a \alpha d(a \beta b+b \beta a)+d(a \beta b+b \beta a) \alpha a+(a \beta b+b \beta a) \alpha d(a) .
\end{aligned}
$$

This implies that

$$
\begin{aligned}
& d(a \alpha a) \beta b+(a \alpha a) \beta d(b)+d(b) \beta(a \alpha a)+b \beta d(a \alpha a)+d(a \alpha b \beta a+a \beta b \alpha a) \\
& =d(a) \alpha a \beta b+d(a) \alpha b \beta a+a \alpha d(a) \beta b+a \alpha a \beta d(b)+a \alpha d(b) \beta a+a \alpha b \beta d(a) \\
& +d(a) \beta b \alpha a+a \beta d(b) \alpha a+d(b) \beta a \alpha a+b \beta d(a) \alpha a+a \beta b \alpha d(a)+b \beta a \alpha d(a) .
\end{aligned}
$$

This implies that

$$
\begin{aligned}
& d(a) \alpha a \beta b+a \alpha d(a) \beta b+a \alpha a \beta d(b)+d(b) \beta a \alpha a+b \beta d(a) \alpha a+b \beta a \alpha d(a)+d(a \alpha b \beta a+a \beta b \alpha a) \\
& =d(a) \alpha a \beta b+d(a) \alpha b \beta a+a \alpha d(a) \beta b+a \alpha a \beta d(b)+a \alpha d(b) \beta a+a \alpha b \beta d(a)+d(a) \beta b \alpha a \\
& +a \beta d(b) \alpha a+d(b) \beta a \alpha a+b \beta d(a) \alpha a+a \beta b \alpha d(a)+b \beta a \alpha d(a) .
\end{aligned}
$$


Now canceling the like terms from both sides we get the required result. Using the condition (*) and since $M$ is 2-torsion free, (iii) follows from (ii). And finally (iv) is obtained by replacing $a+c$ for $a$ in (iii).

Lemma 2.2 Let $U$ be a Lie ideal of a 2-torsion free $\Gamma$-ring $M$ satisfying the condition(*) then $T(U)=\left\{x \in M:[x, M]_{\Gamma} \subseteq U\right\}$ is both a subring and a Lie ideal of $M$ such that $U \subseteq T(U)$.

Proof. We have $U$ is a Lie ideal of $M$, so $[U, M]_{\Gamma} \subseteq U$. Thus $U \subseteq T(U)$. Also we have $[T(U), M]_{\Gamma} \subseteq U \subseteq T(U)$. Hence $T(U)$ is a Lie ideal of $M$. Now suppose that $x, y \in T(U)$ then $[x, m]_{\alpha} \in U \quad$ and $\quad[y, m]_{\alpha} \in U, \forall m \in M \quad$ and $\quad \alpha \in \Gamma$. Now $[x \alpha y, m]_{\beta}=x \alpha[y, m]_{\beta}+[x, m]_{\beta} \alpha y \in U$. Therefore, $[x \alpha y, m]_{\beta} \in U, \forall x, y \in T(U), m \in M$ and $\alpha, \beta \in \Gamma$. Hence $x \alpha y \in T(U)$.

Lemma 2.3 Let $U \nsubseteq Z(M)$ be a Lie ideal of a 2-torsion free semiprime $\Gamma$-ring $M$ satisfying the condition (*), then there exists a nonzero ideal $K=M \Gamma[U, U]_{\Gamma} \Gamma M$ of $M$ generated by $[U, U]_{\Gamma}$ such that $[K, M]_{\Gamma} \subseteq U$.

Proof. First we prove that if $[U, U]_{\Gamma}=0$ then $U \subseteq Z(M)$, so let $[U, U]_{\Gamma}=0$ for $u \in U$ and $\alpha \in \Gamma$, we have $\left[u,[u, x]_{\alpha}\right]_{\alpha}=0, \forall x \in M$. For all $z \in M$ and $\beta \in \Gamma$, we replace $x$ by $x \beta z$ in $\left[u,[u, x]_{\alpha}\right]_{\alpha}=0$ and obtain

$$
\begin{aligned}
0 & =\left[u,[u, x \beta z]_{\alpha}\right]_{\alpha} \\
& =\left[u, x \beta[u, z]_{\alpha}+[u, x]_{\alpha} \beta z\right]_{\alpha} \\
& =\left[u, x \beta[u, z]_{\alpha}\right]_{\alpha}+\left[u,[u, x]_{\alpha} \beta z\right]_{\alpha} \\
& =x \beta\left[u,[u, z]_{\alpha}\right]_{\alpha}+[u, x]_{\alpha} \beta[u, z]_{\alpha}+\left[u,[u, x]_{\alpha}\right]_{\alpha} \beta z+[u, x]_{\alpha} \beta[u, z]_{\alpha} \\
& =2[u, x]_{\alpha} \beta[u, z]_{\alpha}
\end{aligned}
$$

By the 2-torsion freeness of $M$, we obtain $[u, x]_{\alpha} \beta[u, z]_{\alpha}=0$. Now replacing $z$ by $z \gamma x$ , we obtain

$$
\begin{aligned}
0 & =[u, x]_{\alpha} \beta[u, z \gamma x]_{\alpha} \\
& =[u, x]_{\alpha} \beta z \gamma[u, x]_{\alpha}+[u, x]_{\alpha} \beta[u, z]_{\alpha} \gamma x \\
& =[u, x]_{\alpha} \beta z \gamma[u, x]_{\alpha}
\end{aligned}
$$

That is, $[u, x]_{\alpha} \beta M \gamma[u, x]_{\alpha}=0$. Since $M$ is semiprime, $[u, x]_{\alpha}=0$. This implies that $u \in Z(M)$ and therefore, $U \subseteq Z(M)$ is a contradiction. So let $[U, U]_{\Gamma} \neq 0$. Then $K=M \Gamma[U, U]_{\Gamma} \Gamma M$ is a nonzero ideal of $M$ generated by $[U, U]_{\Gamma}$. Let $x, y \in U, m \in M$ and $\alpha, \beta \in \Gamma$, we have $[x, y \beta m]_{\alpha}, y,[x, m]_{\alpha} \in U \subseteq T(U)$.

Hence $[x, y]_{\alpha} \beta m=[x, y \beta m]_{\alpha}-y \beta[x, m]_{\alpha} \in T(U)$. 
Also we can show that, $m \beta[x, y]_{\alpha} \in T(U)$ and therefore, we obtain $\left[[U, U]_{\Gamma}, M\right]_{\Gamma} \subseteq U$. That is, $\left[\left[\left[[x, y]_{\alpha}, m\right]_{\alpha}, s\right]_{\alpha}, t\right]_{\alpha} \in U, \forall m, s, t \in M$ and $\alpha \in \Gamma$.

Hence $\left.\left.[x, y]_{\alpha} \alpha m \alpha s-m \alpha[x, y]_{\alpha} \alpha s+[s, m]_{\alpha} \alpha[x, y]_{\alpha}-\left[s \alpha[x, y]_{\alpha}, m\right]_{\alpha}, m\right]_{\alpha} t\right]_{\alpha} \in T(U)$.

Since $[x, y]_{\alpha} \alpha m \alpha s, s \alpha[x, y]_{\alpha},[s, m]_{\alpha} \alpha[x, y]_{\alpha} \in T(U)$.

Thus we have, $\left[m \alpha[x, y]_{\alpha} \alpha s, t\right]_{\alpha} \in U, \forall m, s, t \in M$ and $\alpha \in \Gamma$. Hence $[K, M]_{\Gamma} \subseteq U$.

Lemma 2.4 Let $U \nsubseteq Z(M)$ be a Lie ideal of a 2-torsion free semiprime $\Gamma$-ring $M$ satisfying the condition (*) and $a \in U$. If $a \alpha U \beta a=\{0\}, \forall \alpha, \beta \in \Gamma$ then $a \alpha a=0$ and there exists a nonzero ideal $K=M[U, U]_{\Gamma} \Gamma M$ of $M$ generated by $[U, U]_{\Gamma}$ such that $[K, M]_{\Gamma} \subseteq U$ and $K \Gamma a=a \Gamma K=\{0\}$.

Proof. If $a \alpha U \beta a=\{0\}, \forall \alpha, \beta \in \Gamma$, then $a \alpha[a, a \delta m]_{\alpha} \beta a=0, \forall m \in M$ and $\delta \in \Gamma$. Thus, by using $(*)$

$$
\begin{aligned}
0 & =a \alpha(a \alpha a \delta m-a \delta m \alpha a) \beta a \\
& =a \alpha a \alpha a \delta m \beta a-a \alpha a \delta m \alpha a \beta a \\
& =a \alpha a \delta a \alpha m-a \alpha a \delta m \beta a \alpha a .
\end{aligned}
$$

Since $a \alpha a \delta a=0$, we have $(a \alpha a) \delta m \beta(a \alpha a)=0$. Since $M$ is semiprime, $a \alpha a=0$. Now we obtain $a \alpha[k \gamma a, m]_{\mu} \alpha u \beta a=0, \forall k \in K, m \in M, u \in U$ and $\alpha, \beta, \mu, \in \Gamma$. Therefore, by using $(*)$ and $a \alpha U \beta a=\{0\}$.

$$
\begin{aligned}
0 & =a \alpha(k \gamma a \mu m-m \mu k \gamma a) \alpha u \beta a \\
& =a \alpha k \gamma a \mu m \alpha u \beta a-a \alpha m \mu k \gamma a \alpha u \beta a \\
& =a \alpha k \gamma a \mu m \beta u \alpha a .
\end{aligned}
$$

So we obtain, $a \alpha k \gamma a \mu m \beta[k, a]_{\gamma} \alpha a=0$. This implies that $a \alpha k \gamma a \mu m \beta(k \gamma a-a \gamma k) \alpha a=0$ and hence $a \alpha k \gamma a \mu m \beta k \gamma a \alpha a-a \alpha k \gamma a \mu m \beta a \gamma k \alpha a=0$. By using (*) and $a \alpha a=0$, we obtain, $(a \alpha k \gamma a) \mu m \beta(a \alpha k \gamma a)=0$. Since $M$ is semiprime, $a \alpha k \gamma a=0$. Thus we find that $(a \alpha k) \Gamma M \beta(a \alpha k)=0$. Hence $a \alpha k=0, \forall k \in K$, that is $a \alpha K=\{0\}$. Similarly we obtain $K \alpha a=\{0\}$.

Lemma 2.5 Let $U \nsubseteq Z(M)$ be a Lie ideal of a 2-torsion free semiprime $\Gamma$-ring $M$ satisfying the condition (*). Let $a, b \in U$ and $\alpha, \beta \in \Gamma$.

(i) If $a \alpha U \beta a=\{0\}$, then $a=0$.

(ii) If $a \alpha U=\{0\}$ (or $U \alpha a=\{0\}$ ), then $a=0$.

(iii) If $u \alpha u \in U, \forall u \in U$ and $a \alpha U \beta b=\{0\}$ then $a \alpha b=0$ and $b \alpha a=0, \forall \alpha \in \Gamma$. 
Proof. (i) By Lemma 2.4, we have $K \alpha a=M \Gamma[U, U]_{\Gamma} \Gamma M \alpha a=\{0\}$ and $a \alpha a=0 \forall \alpha \in \Gamma$.

Therefore, $\forall x, y \in M$ and $\alpha, \beta \in \Gamma$, we obtain:

$$
\begin{aligned}
0 & \left.=\left[[a, x]_{\alpha}, a\right]_{\gamma}\right] \beta y \alpha a \\
& =[a \alpha x-x \alpha a, a]_{\gamma} \beta y \alpha a \\
& =a \alpha[x, a]_{\gamma} \beta y \alpha a-[x, a]_{\gamma} \alpha a \beta y \alpha a \\
& =a \alpha x \gamma a \beta y \alpha a-a \alpha a \gamma x \beta y \alpha a-x \gamma a \alpha a \beta y \alpha a+a \gamma x \alpha a \beta y \alpha a \\
& =a \alpha x \gamma a \beta y \alpha a+a \gamma x \alpha a \beta y \alpha a \\
& =2 a \alpha x \gamma a \beta y \alpha a .
\end{aligned}
$$

By the 2-torsion freeness of $M$, we have $a \alpha x \gamma a \beta y \alpha a=0$. Thus we obtain, $a \alpha x \gamma a \beta y \alpha a \delta x \gamma a=0$. By using (*), we have $(a \alpha x \gamma a) \beta y \delta(a \alpha x \gamma a)=0$. This implies that $(a \alpha x \gamma a) \beta M \delta(a \alpha x \gamma a)=0$. Since $M$ is semiprime $a \alpha x \gamma a=0, \forall x \in M$ and $\alpha, \gamma \in \Gamma$. Again using the semiprimeness of $M$, we obtain $a=0$.

(ii)If $a \alpha U=\{0\}$, then $a \alpha U \beta a=\{0\}), \forall \beta \in \Gamma$, therefore by(i), we obtain $a=0$. Similarly, if $U \alpha a=\{0\}$, then $a=0$.

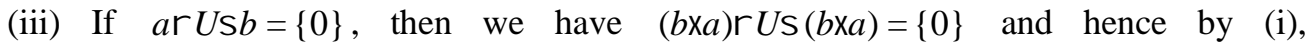
$b \gamma a=0, \forall \gamma \in \Gamma$. Also $(a \gamma b) \alpha U \beta(a \gamma b)=\{0\}$ if $a \alpha U \beta b=\{0\}$ and hence $a \gamma b=0$.

Definition 2.2 Let $M$ be a 2-torsion free semiprime $\Gamma$-ring satisfying the condition(*) and $U$ be a Lie ideal of $M$. Let $D$ be a Jordan derivation on $U$ of $M$. Then $\forall a, b \in U$ and $\alpha \in \Gamma$, we define $G_{\alpha}(a, b)=d(a \alpha b)-d(a) \alpha b-a \alpha d(b)$.

Lemma 2.6 Let $M$ be 2-torsion free semiprime $\Gamma$-ring satisfying the condition (*) and $U$ be a square closed Lie ideal of $M$. Let $d$ be a Jordan derivation on $U$ of $M$. Then $\forall a, b, c \in U$ and $\alpha, \beta \in \Gamma$, the following statements hold.

(i) $G_{\alpha}(a, b)+G_{\alpha}(b, a)=0$;

(ii) $G_{\alpha}(a+b, c)=G_{\alpha}(a, c)+G_{\alpha}(b, c)$;

(iii) $G_{\alpha}(a, b+c)=G_{\alpha}(a, b)+G_{\alpha}(a, c)$;

(iv) $G_{\alpha+\beta}(a, b)=G_{\alpha}(a, b)+G_{\beta}(a, b)$.

Remark 2.1 $d$ is a derivation on $U$ of $M$ if and only if $G_{\alpha}(a, b)=0, \forall a, b \in U$ and $\alpha \in \Gamma$.

Remark 2.2 If $U$ is a commutative Lie ideal of $M$, then by Lemma $2.3 U \subseteq Z(M)$. In this situation, we have seen that $G_{\alpha}(a, b)=0, \forall a, b \in U$ and $\alpha \in \Gamma$ which is obtained from Lemma 2.1(i). So, we proved our main result for admissible Lie ideal of $M$.

Lemma 2.7 Let $M$ be 2-torsion free $\Gamma$-ring satisfying the condition $(*)$ and $U$ be a square closed Lie ideal of $M$. If $d$ is a Jordan derivation on $U$ of $M$. Then 
$(i) G_{\alpha}(a, b) \beta u \gamma[a, b]_{\alpha}+[a, b]_{\alpha} \beta u \gamma G_{\alpha}(a, b)=0, \forall a, b, u \in U \quad$ and $\alpha, \beta \in \Gamma$

(ii) $G_{\alpha}(a, b) \alpha u \alpha[a, b]_{\alpha}+[a, b]_{\alpha} \alpha u \alpha G_{\alpha}(a, b)=0, \forall a, b, u \in U$ and $\alpha, \beta \in \Gamma$

(iii) $G_{\alpha}(a, b) \beta u \beta[a, b]_{\alpha}+[a, b]_{\alpha} \beta u \beta G_{\alpha}(a, b)=0, \forall a, b, u \in U$ and $\alpha, \beta \in \Gamma$.

Proof. (i) For any $a, b, u \in U$ and $\alpha, \beta, \gamma \in \Gamma$, we have by using Lemma 2.1(iv)

$$
\begin{aligned}
W & =d(4 a \alpha b \beta u \gamma b \alpha a+4 b \alpha a \beta u \gamma a \alpha b) \\
& =d((2 a \alpha b) \beta u \gamma(2 b \alpha a)+(2 b \alpha a) \beta u \gamma(2 a \alpha b)) \\
& =4 d(a \alpha b) \beta u \gamma b \alpha a+4 a \alpha b \beta d(u) \gamma b \alpha a+4 a \alpha b \beta u \gamma d(b \alpha a) \\
& +4 d(b \alpha a) \beta u \gamma a \alpha b+4 b \alpha a \beta d(u) \gamma a \alpha b+4 b \alpha a \beta u \gamma d(a \alpha b) .
\end{aligned}
$$

On the other hand by using Lemma 2.1(iii)

$$
\begin{aligned}
W & =d(a \alpha(4 b \beta u \gamma b) \alpha a+b \alpha(4 a \beta u \gamma a) \alpha b) \\
& =d(a \alpha(4 b \beta u \gamma b) \alpha a)+d(b \alpha(4 a \beta u \gamma a) \alpha b) \\
& =4 d(a) \alpha b \beta u \gamma b \alpha a+4 a \alpha d(b \beta u \gamma b) \alpha a+4 a \alpha b \beta u \gamma b \alpha d(a)+4 d(b) \alpha a \beta u \gamma a \alpha b \\
& +4 b \alpha d(a \beta u \gamma a) \alpha b+4 b \alpha a \beta u \gamma a \alpha d(b) \\
& =4(d(a) \alpha b \beta u \gamma b \alpha a+a \alpha d(b) \beta u \gamma b \alpha a+a \alpha b \beta d(u) \gamma b \alpha a+a \alpha b \beta u \gamma d(b) \alpha a \\
& +a \alpha b \beta u \gamma b \alpha d(a)+d(b) \alpha a \beta u \gamma a \alpha b+b \alpha d(a) \beta u \gamma a \alpha b+b \alpha a \beta d(u) \gamma a \alpha b \\
& +b \alpha a \beta u \gamma d(a) \alpha b+b \alpha a \beta u \gamma a \alpha d(b)) .
\end{aligned}
$$

Equating two expressions for $W$ and canceling the like terms from both sides, we get

$4(d(a \alpha b) \beta u \gamma b \alpha a+a \alpha b \beta u \gamma d(b \alpha a)+d(b \alpha a) \beta u \gamma a \alpha b+b \alpha a \beta u \gamma d(a \alpha b))$

$=4(d(a) \alpha b \beta u \gamma b \alpha a+a \alpha d(b) \beta u \gamma b \alpha a+a \alpha b \beta u \gamma d(b) \alpha a+a \alpha b \beta u \gamma b \alpha d(a)$

$+d(b) \alpha a \beta u \gamma a \alpha b+b \alpha d(a) \beta u \gamma a \alpha b+b \alpha a \beta u \gamma d(a) \alpha b+b \alpha a \beta u \gamma a \alpha d(b))$.

This gives

$4(d(a \alpha b) \beta u \gamma b \alpha a-d(a) \alpha b \beta u \gamma b \alpha a-a \alpha d(b) \beta u \gamma b \alpha a+d(b \alpha a) \beta u \gamma a \alpha b$

$-d(b) \alpha a \beta u \gamma a \alpha b-b \alpha d(a) \beta u \gamma a \alpha b+a \alpha b \beta u \gamma d(b \alpha a)-a \alpha b \beta u \gamma d(b) \alpha a$

$-a \alpha b \beta u \gamma b \alpha d(a)+b \alpha a \beta u \gamma d(a \alpha b)-b \alpha a \beta u \gamma d(a) \alpha b-b \alpha a \beta u \gamma a \alpha d(b))=0$.

This implies that

$$
\begin{aligned}
& 4((d(a \alpha b)-d(a) \alpha b-a \alpha d(b)) \beta u \gamma b \alpha a+(d(b \alpha a)-d(b) \alpha a-b \alpha d(a)) \beta u \gamma a \alpha b+ \\
& a \alpha b \beta u \gamma(d(b \alpha a)-d(b) \alpha a-b \alpha d(a))+b \alpha a \beta u \gamma(d(a \alpha b)-d(a) \alpha b-a \alpha d(b)))=0 .
\end{aligned}
$$

Now using the Definition 2.2 and 2-torsion freeness of $M$, we obtain

$$
G_{\alpha}(a, b) \beta u \gamma b \alpha a+G_{\alpha}(b, a) \beta u \gamma a \alpha b+a \alpha b \beta u \gamma G_{\alpha}(b, a)+b \alpha a \beta u \gamma G_{\alpha}(a, b)=0 .
$$

This implies that

$$
G_{\alpha}(a, b) \beta u \gamma[a, b]_{\alpha}+[a, b]_{\alpha} \beta u \gamma G_{\alpha}(a, b)=0, \forall a, b, u \in U, \alpha, \beta, \gamma \in \Gamma .
$$


If we consider

$$
W=d(4 a \alpha b \alpha u \alpha b \alpha a+4 b \alpha a \alpha u \alpha a \alpha b) \text { and } W=d(4 a \alpha b \beta u \beta b \alpha a+4 b \alpha a \beta u \beta a \alpha b)
$$

for (ii) and (iii) respectively and proceeding in the same way as in the proof of (i) by the similar arguments, we get (ii) and (iii).

Lemma 2.8 Let $U$ be an admissible Lie ideal of a 2-torsion free semiprime $\Gamma$-ring $M$ and let $a, b \in U$. If $a \alpha u \beta b+b \alpha u \beta a=0, \forall u \in U, \alpha, \beta \in \Gamma$, then $a \alpha u \beta b=0=b \alpha u \beta a$.

Proof. Let $x \in U$ and $\gamma \in \Gamma$ be any elements. Using the relation $a \alpha u \beta b+b \alpha u \beta a=0, \forall u \in U, \alpha, \beta \in \Gamma$ repeatedly, we get

$$
\begin{aligned}
4(a \alpha u \beta b) \gamma x \gamma(a \alpha u \beta b) & =-4(b \alpha u \beta a) \gamma x \gamma(a \alpha u \beta b) \\
& =-(b \alpha(4 u \beta a \gamma x) \gamma a) \alpha u \beta b \\
& =(a \alpha(4 u \beta a \gamma x) \gamma b) \alpha u \beta b \\
& =a \alpha u \beta(4 a \gamma x \gamma b) \alpha u \beta b \\
& =-a \alpha u \beta(4 b \gamma x \gamma a) \alpha u \beta b \\
& =-4(a \alpha u \beta b) \gamma x \gamma(a \alpha u \beta b) .
\end{aligned}
$$

This implies, $8((a \alpha u \beta b) \gamma x \gamma(a \alpha u \beta b))=0$. Since $M$ is 2-torsion free, $(a \alpha u \beta b) \gamma x \gamma(a \alpha u \beta b)=0$. Therefore, $(a \alpha u \beta b) \gamma U \gamma(a \alpha u \beta b)=0$. Thus by Lemma 2.5, we get $a \alpha u \beta b=0$. Similarly, it can be shown that $b \alpha u \beta a=0$.

Corollary 2.1 Let $M$ be a 2-torsion free semiprime $\Gamma$-ring satisfying the condition(*) and $U$ be an admissible Lie ideal of $M$. If $d$ is a Jordan derivation on $U$ of $M$. Then $\forall a, b, u \in U, \alpha, \beta, \gamma \in \Gamma$

$$
\begin{aligned}
& (i) G_{\alpha}(a, b) \beta u \gamma[a, b]_{\alpha}=0 ;(i i)[a, b]_{\alpha} \beta u \gamma G_{\alpha}(a, b)=0 ;(i i i) G_{\alpha}(a, b) \alpha u \alpha[a, b]_{\alpha}=0 ; \\
& (i v)[a, b]_{\alpha} \alpha u \alpha G_{\alpha}(a, b)=0 ;(v) G_{\alpha}(a, b) \beta u \beta[a, b]_{\alpha}=0 ;(v i)[a, b]_{\alpha} \beta u \beta G_{\alpha}(a, b)=0 .
\end{aligned}
$$

Proof. Applying the result of Lemma 2.8 in that of Lemma 2.7, we obtain these results.

Lemma 2.9 Let $M$ be a 2-torsion free semiprime $\Gamma$-ring satisfying the condition (*) and $U$ be an admissible Lie ideal of $M$. Let $d$ be a Jordan derivation on $U$ of $M$. Then $\forall a, b, u \in U, \alpha, \beta, \gamma \in \Gamma$

$$
\begin{aligned}
& \text { (i) } G_{\alpha}(a, b) \beta u \beta[x, y]_{\alpha}=0 ;(i i)[x, y]_{\alpha} \beta u \beta G_{\alpha}(a, b)=0 \\
& \text { (iii) } G_{\alpha}(a, b) \beta u \beta[x, y]_{\gamma}=0 ;(i v)[x, y]_{\gamma} \beta u \beta G_{\alpha}(a, b)=0
\end{aligned}
$$

Proof. (i) If we substitute $a+x$ for $a$ in the Corollary 2.1 (v), we get

$$
G_{\alpha}(a+x, b) \beta u \beta[a+x, b]_{\alpha}=0
$$

This implies

$$
G_{\alpha}(a, b) \beta u \beta[a, b]_{\alpha}+G_{\alpha}(a, b) \beta u \beta[x, b]_{\alpha}+G_{\alpha}(x, b) \beta u \beta[a, b]_{\alpha}+G_{\alpha}(x, b) \beta u \beta[x, b]_{\alpha}=0 .
$$


Thus by using Corollary 2.1 (v), we have

$$
G_{\alpha}(a, b) \beta u \beta[x, b]_{\alpha}+G_{\alpha}(x, b) \beta u \beta[a, b]_{\alpha}=0 .
$$

Thus, we obtain

$$
\begin{aligned}
& \left(G_{\alpha}(a, b) \beta u \beta[x, b]_{\alpha}\right) \beta u \beta\left(G_{\alpha}(a, b) \beta u \beta[x, b]_{\alpha}\right) \\
& =-G_{\alpha}(a, b) \beta u \beta[x, b]_{\alpha} \beta u \beta G_{\alpha}(x, b) \beta u \beta[a, b]_{\alpha}=0 .
\end{aligned}
$$

Hence, by Lemma 2.5(i), we get

$$
G_{\alpha}(a, b) \beta u \beta[x, b]_{\alpha}=0
$$

Similarly, by replacing $b+y$ for $b$ in this result, we get

$$
G_{\alpha}(a, b) \beta u \beta[x, y]_{\alpha}=0
$$

(ii) Proceeding in the same way as described above by the similar replacements successively in Corollary 2.1 (vi), we obtain

$$
[x, y]_{\gamma} \beta u \beta G_{\alpha}(a, b)=0, \forall a, b, x, y, u \in U, \alpha, \beta \in \Gamma
$$

(iii) Replacing $\alpha+\gamma$ for $\alpha$ in (i), we get

$$
G_{\alpha+\gamma}(a, b) \beta u \beta[x, y]_{\alpha+\gamma}=0
$$

This implies

$$
\left(G_{\alpha}(a, b)+G_{\gamma}(a, b)\right) \beta u \beta\left([x, y]_{\alpha}+[x, y]_{\gamma}\right)=0
$$

Therefore

$$
G_{\alpha}(a, b) \beta u \beta[x, y]_{\alpha}+G_{\alpha}(a, b) \beta u \beta[x, y]_{\gamma}+G_{\gamma}(a, b) \beta u \beta[x, y]_{\alpha}+G_{\gamma}(a, b) \beta u \beta[x, y]_{\gamma}=0 .
$$

Thus by using Corollary 2.1 (vi), we get

$$
G_{\alpha}(a, b) \beta u \beta[x, y]_{\gamma}+G_{\gamma}(a, b) \beta u \beta[x, y]_{\alpha}=0 .
$$

Thus, we obtain

$$
\begin{aligned}
& \left(G_{\alpha}(a, b) \beta u \beta[x, y]_{\gamma}\right) \beta u \beta\left(G_{\alpha}(a, b) \beta u \beta[x, y]_{\gamma}\right) \\
& =-G_{\alpha}(a, b) \beta u \beta[x, y]_{\gamma} \beta u \beta G_{\gamma}(a, b) \beta u \beta[x, y]_{\alpha}=0 .
\end{aligned}
$$

Hence, by Lemma 2.5(i), we obtain $G_{\alpha}(a, b) \beta u \beta[x, y]_{\gamma}=0$.

(iv) As in the proof of (iii), the similar replacement in (ii) produces (iv).

Lemma 2.10 ([8], Lemma 3.6.1) Every semiprime $\Gamma$-ring contains no nonzero nilpotent ideal.

Corollary 2.2 ([8], Corollary 3.6.2) Semiprime $\Gamma$-ring has no nonzero nilpotent element.

Lemma 2.11 ([8], Lemma 3.6.2) The center of a semiprime $\Gamma$-ring does not contain any nonzero nilpotent element. 
Theorem 2.1 Let $M$ be a 2-torsion free semiprime $\Gamma$-ring satisfying the condition(*) and $U$ be an admissible Lie ideal of $M$. If $d: M \rightarrow M$ is a Jordan derivation on $U$, then $d$ is a derivation on $U$ of $M$.

Proof. Let $a, b, y, u \in U$ and $\alpha, \beta \in \Gamma$. Then by Lemma 2.9 (iii), we get

$$
\begin{aligned}
& 2\left[G_{\alpha}(a, b), y\right]_{\beta} \beta u \beta\left[G_{\alpha}(a, b), y\right]_{\beta}=2\left(G_{\alpha}(a, b) \beta y-y \beta G_{\alpha}(a, b)\right) \beta u \beta\left[G_{\alpha}(a, b), y\right]_{\beta} \\
& =G_{\alpha}(a, b) \beta(2 y \beta u) \beta\left[G_{\alpha}(a, b), y\right]_{\beta}-2 y \beta G_{\alpha}(a, b) \beta u \beta\left[G_{\alpha}(a, b), y\right]_{\beta}=0 .
\end{aligned}
$$

Since $2 y \beta u \in U$ and $G_{\alpha}(a, b) \in M, \forall a, b, y, u \in U$ and $\alpha, \beta \in \Gamma$. Hence, by Lemma 2.5(i), $\quad\left[G_{\alpha}(a, b), y\right]_{\beta}=0$, where $G_{\alpha}(a, b) \in M, \forall a, b, y \in U$ and $\alpha, \beta \in \Gamma$. Therefore, $G_{\alpha}(a, b) \in Z(M)$, the center of $M$. Now, let $x \in U$ and $\gamma, \delta \in \Gamma$. Then, we have $G_{\alpha}(a, b) \gamma[x, y]_{\alpha} \delta u \delta G_{\alpha}(a, b) \gamma[x, y]_{\alpha}=0$, by using Lemma 2.9(ii).

Thus by using Lemma 2.5(i)

$$
G_{\alpha}(a, b) \gamma[x, y]_{\alpha}=0 .
$$

Similarly, we get $[x, y]_{\alpha} \gamma G_{\alpha}(a, b) \delta u \delta[x, y]_{\alpha} \gamma G_{\alpha}(a, b)=0$, by using Lemma 2.9(i).

Now by Lemma 2.5(i), we get

$$
[x, y]_{\alpha} \gamma G_{\alpha}(a, b)=0 .
$$

Similarly, by Lemma 2.9(iv), we have $G_{\alpha}(a, b) \gamma[x, y]_{\beta} \delta u \delta G_{\alpha}(a, b) \gamma[x, y]_{\beta}=0$. By Lemma 2.5(i), it follows that

$$
G_{\alpha}(a, b) \gamma[x, y]_{\beta}=0 .
$$

Also, by Lemma 2.9(iii), we have $[x, y]_{\beta} \gamma G_{\alpha}(a, b) \delta u \delta[x, y]_{\beta} \gamma G_{\alpha}(a, b)=0$. Hence by Lemma 2.5(i), we get

$$
[x, y]_{\beta} \gamma G_{\alpha}(a, b)=0 .
$$

Thus, we have

$$
\begin{aligned}
2 G_{\alpha}(a, b) \gamma G_{\alpha}(a, b) & =G_{\alpha}(a, b) \gamma\left(G_{\alpha}(a, b)+G_{\alpha}(a, b)\right) \\
& =G_{\alpha}(a, b) \gamma\left(G_{\alpha}(a, b)-G_{\alpha}(b, a)\right) \\
& =G_{\alpha}(a, b) \gamma(d(a \alpha b)-d(a) \alpha b-a \alpha d(b)-d(b \alpha a)+d(b) \alpha a+b \alpha d(a)) \\
& =G_{\alpha}(a, b) \gamma(d(a \alpha b-b \alpha a)+(b \alpha d(a)-d(a) \alpha b)+(d(b) \alpha a-a \alpha d(b))) \\
& =G_{\alpha}(a, b) \gamma\left(d\left([a, b]_{\alpha}\right)+[b, d(a)]_{\alpha}+[d(b), a]_{\alpha}\right) \\
& =G_{\alpha}(a, b) \gamma d\left([a, b]_{\alpha}\right)-G_{\alpha}(a, b) \gamma[d(a), b]_{\alpha}-G_{\alpha}(a, b) \gamma[a, d(b)]_{\alpha} .
\end{aligned}
$$

Since $d(a), d(b) \in M$, so by using Lemma 2.9(i), we get

$$
G_{\alpha}(a, b) \gamma[d(a), b]_{\alpha}=G_{\alpha}(a, b) \gamma[a, d(b)]_{\alpha}=0 .
$$


Thus

$$
2 G_{\alpha}(a, b) \gamma G_{\alpha}(a, b)=G_{\alpha}(a, b) \gamma d\left([a, b]_{\alpha}\right) .
$$

Adding (3) and (4), we obtain $G_{\alpha}(a, b) \gamma[x, y]_{\beta}+[x, y]_{\beta} \gamma G_{\alpha}(a, b)=0$. Then by Lemma 2.1 (i) with the use of (3), we have

$$
\begin{aligned}
0 & =d\left(G_{\alpha}(a, b) \gamma[x, y]_{\beta}+[x, y]_{\beta} \gamma G_{\alpha}(a, b)\right) \\
& =d\left(G_{\alpha}(a, b)\right) \gamma[x, y]_{\beta}+d\left([x, y]_{\beta}\right) \gamma G_{\alpha}(a, b)+G_{\alpha}(a, b) \gamma d\left([x, y]_{\beta}\right)+[x, y]_{\beta} \gamma d\left(G_{\alpha}(a, b)\right) \\
& =d\left(G_{\alpha}(a, b)\right) \gamma[x, y]_{\beta}+2 G_{\alpha}(a, b) \gamma d\left([x, y]_{\beta}\right)+[x, y]_{\beta} \gamma d\left(G_{\alpha}(a, b)\right) .
\end{aligned}
$$

Since $G_{\alpha}(a, b) \in Z(M)$ and therefore $d\left([x, y]_{\beta}\right) \gamma G_{\alpha}(a, b)=G_{\alpha}(a, b) \gamma d\left([x, y]_{\beta}\right)$.

Hence, we get

$$
2 G_{\alpha}(a, b) \gamma d\left([x, y]_{\beta}\right)=-d\left(G_{\alpha}(a, b)\right) \gamma[x, y]_{\beta}-[x, y]_{\beta} \gamma d\left(G_{\alpha}(a, b)\right) .
$$

Then from (5) and (6) we have

$$
\begin{aligned}
4 G_{\alpha}(a, b) \gamma G_{\alpha}(a, b) & =2 G_{\alpha}(a, b) \gamma d\left([a, b]_{\alpha}\right) \\
& =-d\left(G_{\alpha}(a, b)\right) \gamma[a, b]_{\alpha}-[a, b]_{\alpha} \gamma d\left(G_{\alpha}(a, b)\right) .
\end{aligned}
$$

Thus we obtain

$$
\begin{aligned}
& 4 G_{\alpha}(a, b) \gamma G_{\alpha}(a, b) \gamma G_{\alpha}(a, b) \gamma G_{\alpha}(a, b)= \\
& -G_{\alpha}(a, b) \gamma d\left(G_{\alpha}(a, b)\right) \gamma[a, b]_{\alpha} \gamma G_{\alpha}(a, b)-G_{\alpha}(a, b) \gamma[a, b]_{\alpha} \gamma d\left(G_{\alpha}(a, b)\right) \gamma G_{\alpha}(a, b) .
\end{aligned}
$$

Since by (1) and (2), $G_{\alpha}(a, b) \gamma[a, b]_{\alpha} \gamma d\left(G_{\alpha}(a, b)\right) \gamma G_{\alpha}(a, b)=0$ and

$$
G_{\alpha}(a, b) \gamma d\left(G_{\alpha}(a, b)\right) \gamma[a, b]_{\alpha} \gamma G_{\alpha}(a, b)=0 .
$$

So, we get

$$
4 G_{\alpha}(a, b) \gamma G_{\alpha}(a, b) \gamma G_{\alpha}(a, b) \gamma G_{\alpha}(a, b)=0 .
$$

Therefore, $4\left(G_{\alpha}(a, b) \gamma\right)^{3} G_{\alpha}(a, b)=0$. Since $M$ is 2-torsion free, so we have

$$
\left(G_{\alpha}(a, b) \gamma\right)^{3} G_{\alpha}(a, b)=0
$$

But, it follows that $G_{\alpha}(a, b)$ is a nilpotent element of the $\Gamma$-ring $M$. Since by Lemma 2.11 , the center of a semiprime $\Gamma$-ring does not contain any nonzero nilpotent element, so we get $G_{\alpha}(a, b)=0, \forall a, b \in U$ and $\alpha \in \Gamma$. It means that, $d$ is a derivation on $U$ of $M$.

\section{REFERENCES}

[1] Ashraf , M. and Rehman, N., On Lie ideals and Jordan left derivations of prime rings, Arch. Math.(Brno), 36 (2000), 201-206.

[2] Awtar, R., Lie ideals and Jordan derivations of prime rings, Amer.Math.Soc. 90(1) (1984), 9-14. 
[3] Barnes, W. E., On the Г-rings of Nobusawa, Pacific J. Math., 18(1966), 411-422.

[4] Bergen, J., Herstein, I. N. and Kerr ,J. W., Lie ideals and derivations of prime rings, J. Algebra, 71(1981), 259-267.

[5] Bresar, M., Jordan derivations on semiprime rings, Proc. Amer. Math. Soc., 104(4), (1988) 10031004.

[6] Ceven, Y., Jordan left derivations on completely prime gamma rings, C. U. Fen-Edebiyat Fakultesi Fen Bilimleri Dergisi 23(2002), 39-43.

[7] Chakraborty, S. and Paul, A. C., On Jordan k-derivations of 2-torsion free Prime $\Gamma_{N}$-rings, Punjab Univ. J. Math., 40(2008), 97-101.

[8] Chakraborty, S. and Paul, A. C., k-derivations and k-homomorphisms of $\Gamma$-rings, Lap Lambert Academic Publishing GmbH \& Co. KG, Geometry, 2012.

[9] Haetinger, C., Higher derivations on Lie ideals, Tenclencias em Matematica Aplicada e. computacional, 3(1) (2002), 141-145.

[10] Halder, A.K. and Paul, A. C., Jordan left derivations on Lie ideals of prime $\Gamma$-rings, Punjab University J.Math.,(2011), 01-07.

[11] Herstein, I. N., Jordan derivations of prime rings, Proc. Amer. Math. Soc., 8(1957), 1104-1110.

[12] Herstein, I. N., Topics in Ring Theory, (Ed. The University of Chicago Press, Chicago), (1969).

[13] Hongan, M., Rehman, N. and Al Omary, R. M., Lie ideals and Jordan triple derivations of rings, Rend. Sem. Mat. Univ. Padova., 125, (2011), 147-156.

[14] Lanski, C. and Montgomery, S., Lie structure of prime rings of characteristic 2, Pacific J. Math., 42, No. 1 (1972), 117-136.

[15] Nobusawa, N., On the generalizeation of the ring theory, Osaka J. Math. 1(1964), 81-89.

[16] Sapanci, M. and Nakajima, A., Jordan Derivations on Completely Prime $\Gamma$-Rings, Math. Japonica 46, (1997), 47-51. 